\title{
The CanX-7 ADS-B Mission: Signal Propagation Assessment
}

\author{
Ron Vincent ${ }^{1}$, Kelly Freitag ${ }^{2}$ \\ ${ }^{1}$ Department of Physics and Space Science, Royal Military College of Canada, Kingston, Canada \\ ${ }^{2}$ Royal Canadian Air Force W/C William G. Barker VC Aerospace College, Winnipeg, Canada \\ Email: Ron.Vincent@rmc.ca
}

How to cite this paper: Vincent, R. and Freitag, K. (2019) The CanX-7 ADS-B Mission: Signal Propagation Assessment. Positioning, 10, 1-15.

https://doi.org/10.4236/pos.2019.101001

Received: January 20, 2019

Accepted: February 25, 2019

Published: February 28, 2019

Copyright (c) 2019 by author(s) and Scientific Research Publishing Inc. This work is licensed under the Creative Commons Attribution International License (CC BY 4.0).

http://creativecommons.org/licenses/by/4.0/

\begin{abstract}
The CanX-7 Automatic Dependent Surveillance-Broadcast (ADS-B) nanosatellite mission collected more than four million ADS-B messages between October 2016 and April 2017. An analysis of data collected over the north Atlantic Ocean from 05 to 28 Oct included 20,707 position messages in which the angle from satellite nadir to aircraft was determined. The proximity of the received signal strength to the noise floor of the sensor allowed for an analysis of optimal aircraft-satellite orientation for ADS-B transmission detection. The results showed a significant disparity between descending and ascending passes of the satellite. For descending passes, the average nadir angle was $50.1^{\circ}$ with $90 \%$ of the contacts greater than $40^{\circ}$. The ascending passes had an average nadir angle of $31.6^{\circ}$ with only $24.8 \%$ of the contacts exceeding $40^{\circ}$. The evidence suggests that the satellite magnetic torquer may not have been fully aligned with the north magnetic pole as the satellite moved northward, resulting in ascending pass nadir angles that were not reflective of the full range of values. Further analysis of the descending passes showed agreement with an ADS-B signal propagation model with peak reception at nadir angles of $51^{\circ} \pm 8^{\circ}$. For space-based ADS-B operations, the results support the replacement of the current aircraft upper quarter-wave monopole to an antenna that will transmit more energy directly above the airframe.
\end{abstract}

\section{Keywords}

ADS-B, Space-Based ADS-B, CanX-7, Air Traffic Control

\section{Introduction}

Automatic Dependent Surveillance-Broadcast (ADS-B) is a technology in which aircraft transmit identification, position, velocity and status on $1090 \mathrm{MHz}$. The system uses a global positioning system (GPS) receiver, to determine the air- 
craft's position, which is transmitted along with aircraft identification, velocity, and status in a coded message. The 120-bit ADS-B message is broadcast at random intervals between 0.4 and 0.6 seconds to mitigate signal collisions between aircraft. The use of GPS positioning and the frequent update cycle allow surveillance information that is more accurate and timelier than conventional surveillance radars. Signal power ranges between 75 and $500 \mathrm{~W}$ depending on aircraft category with the vertically polarized signal alternating between top- and bottom-mounted quarter-wave monopole antennas [1].

The detection of ADS-B transmissions from space will allow air traffic monitoring around the globe, a significant increase from the current $30 \%$ global coverage, which shall enhance safety of flight [2]. Precise positioning provided by space-based ADS-B will allow more economical routing of air traffic in remote and oceanic airspace by reducing the required spacing between transiting aircraft, which will lessen both fuel costs and greenhouse emissions. The dawn of space-based air traffic control has begun with the launch of the Iridium NEXT constellation between 2017 and 2019, consisting of 75 satellites (66 operational and 9 spares) in low Earth orbit equipped with ADS-B receivers. In April 2019 the system was put into trial use over the North Atlantic, which is the busiest oceanic airspace in the world with over 50,000 flights per year [2]. The constellation is expected to provide worldwide tracking of ADS-B equipped aircraft in the next few years.

The first demonstration of the reception of ADS-B signals from near space was carried out by the Royal Military College of Canada (RMC) in 2009 with the Flying Laboratory for the Observation of ADS-B Transmissions (FLOAT), a high altitude balloon mission that tracked aircraft from stratospheric altitudes [3] [4]. The success of the initial FLOAT mission led to further research of ADS-B signal propagation, including the determination of electron content in the ionosphere as a function of Faraday rotation [5] [6] [7] and in-depth analysis of ADS-B transmissions from aircraft to satellite [8] [9] [10] [11]. RMC also developed an ADS-B payload designed to fly on a CubeSat platform [12] that was integrated onto CanX-7, a $3 \mathrm{U}(10 \times 10 \times 34 \mathrm{~cm})$ nanosatellite built and operated by the University of Toronto Institute of Aerospace Studies-Space Flight Laboratory. CanX-7 was launched into a $690 \mathrm{~km}$ sun-synchronous orbit on September 2016 and collected ADS-B data for seven months prior to deploying a drag sail in May 2017. During the mission, the payload received approximately 4.3 million ADS-B messages (Figure 1). As a result of the magnetic torquer attitude control system, which aligns the satellite with the Earth's magnetic field, CanX-7 could only point the ADS-B antenna downward in one hemisphere during an orbit. As a result, all signals were collected in the Northern Hemisphere, except for November when the polarity of the magnetic torquer was reversed for one month.

A preliminary analysis of the CanX-7 mission [13] indicated that an ADS-B signal propagation model [10] was consistent with the satellite data. This paper gives a detailed analysis of ADS-B signal propagation from aircraft to satellite. The results indicate that ADS-B transmissions are strongest at high off-nadir 


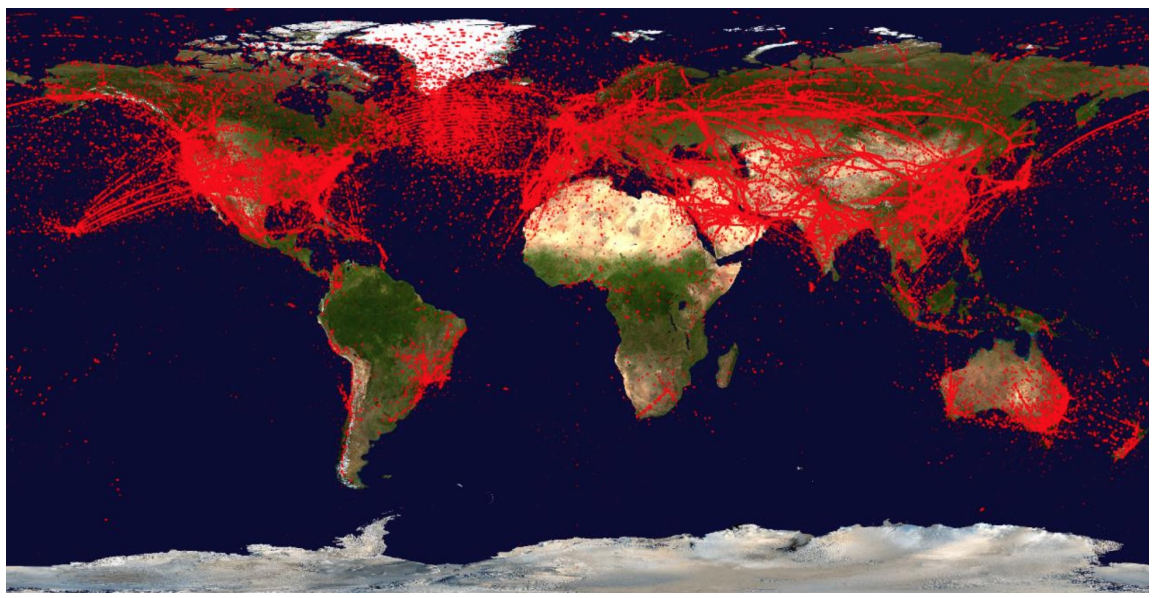

Figure 1. Composite of aircraft position messages received by the ADS-B payload on CanX-7, October 2016 to April 2017 [14].

angles as a result of the aircraft antenna radiation pattern. This is significant for nadir pointing ADS-B receivers, implying that the aircraft quarter-wave monopole is not ideal for space applications. Section 2 of this paper describes methods used for the analysis, Section 3 contains the results and Section 4 gives a summary and conclusion.

\section{Methods}

Data collection and analysis presented in this section represent a rare description of observed space-based ADS-B propagation. The CubeSat pointing mechanism resulted in findings that differed depending on the direction of travel of the satellite.

\subsection{Data Collection}

The period of observation for this analysis extends from 05 October to 28 October 2016 over the Shanwick and Gander Oceanic Control Areas in the North Atlantic. The area of operation (AO) was chosen because it contains the North Atlantic Organized Track System (NAT-OTS), which handles approximately 1,400 flights a day, and is relatively quiet with respect to $1090 \mathrm{MHz}$ transmissions not related to ADS-B. During the observation period, CanX-7 passed through the middle of the AO at approximately 1230 UTC daily for north-tosouth descending passes and at approximately 2300 UTC for south-to-north ascending passes. Typically, there were two additional passes per day, one descending and one ascending, that flew over the eastern or western edges of the AO (Figure 2). With a speed of $7.5 \mathrm{~km} / \mathrm{s}$, the satellite took approximately five minutes to pass over the AO. Air traffic in the region typically experiences two peaks every day, with an eastward flow at 0300 UTC and a westward flow at 1400 UTC [10]. Lulls in air traffic occur at 0900 UTC and 2300 UTC. This daily pattern resulted in significantly more aircraft available for detection during descending passes than ascending passes. The database for this study included 90 


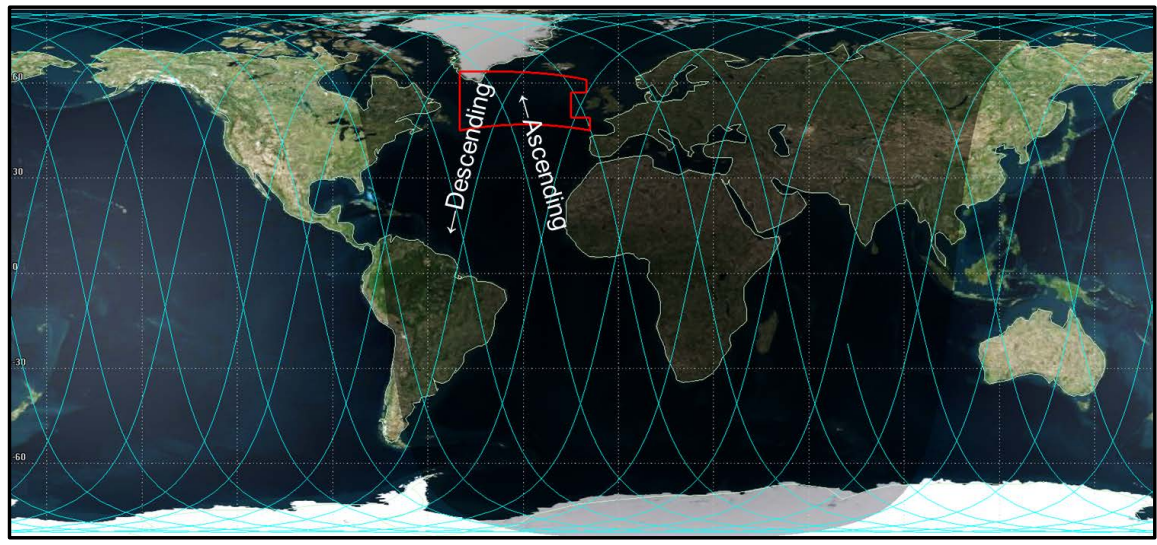

Figure 2. CanX-7 ground track for a typical 24-hour period with descending and ascending passes is shown over the AO highlighted in red [13]. The ground tracks move westward each day, resulting in coverage over the central portion of the AO for two passes and peripheral coverage for two additional passes. Image created with Systems Toolkit Software (STK), from Analytical Graphics Incorporated (AGI).

passes through the AO, representing 20,707 aircraft positions from 1559 contacts and 813 unique aircraft. Air traffic transited predominantly west or east (93\%) as aircraft followed the NAT-OTS between Europe and North America. A smaller number of contacts traveled north or south (7\%), mostly between Africa and Europe through the eastern portion of the AO. Table 1 contains a summary of data collection statistics.

\subsection{Data Analysis}

The power of the signal received by the satellite $\left(P_{r}\right)$ is described in Equation (1),

$$
P_{r}=\frac{P_{t} G_{t} G_{r}}{L_{a}}\left(\frac{\lambda}{4 d \pi}\right)
$$

where $P_{t}$ is transmitted power, $G_{t}$ is transmitter gain, $G_{r}$ is receiver gain, $L_{a}$ represents atmospheric losses, $\lambda$ is the wavelength of the ADS-B signal, and $d$ is the distance between the aircraft and satellite. Table 2 summarizes the values of the variables in Equation (1) for the CanX-7 mission. Assuming $500 \mathrm{~W}$ maximum power for trans-Atlantic airliners and negligible atmospheric losses, the main factors that affect the received signal strength is the distance from the aircraft to the satellite and the radiation patterns of the transmitting and receiving antennas. The satellite receiver has a broad beam width with relatively homogeneous gain, while the quarter wave monopole transmitters on the aircraft have a flattened donut-shaped pattern, with no power transmitted directly above the antenna. Aircraft have transmitting antennas mounted top and bottom on the aircraft. Reflections of ADS-B signals off the ocean from the bottom antenna are too weak for detection and may be ignored [11]. The radiation pattern of the upper antenna is modified by aircraft structures, resulting in the highest gain observed at lower elevation angles [10]. Figure 3 shows that the modeled received power occurs at $51^{\circ} \pm 8^{\circ}$ measured from the satellite nadir to the aircraft. 
Table 1. ADS-B Mission Statistics for the Northern Hemisphere, 04 to 31 October 2016.

\begin{tabular}{cc}
\hline Property & Value \\
\hline Passes & 90 \\
ADS-B Position Messages & 20,707 \\
Unique Aircraft & 813 \\
Descending Pass Messages & 14,302 \\
Ascending Pass Messages & 6405 \\
East-West Traffic & $64 \%$ East, 29\% West \\
North-South Traffic & 6\% North, 1\% South \\
\hline
\end{tabular}

Table 2. Description of variables for signal strength received by CanX-7 ADS-B payload.

\begin{tabular}{cc}
\hline Property & Description \\
\hline Transmitter Power & $500 \mathrm{~W}$ maximum $[1]$ \\
Transmitter Antenna & Quarter wave monopole, vertical polarization \\
Transmitter Gain & Typically $5.1 \mathrm{~dB}$ maximum \\
Receiver Antenna & Patch, Right-hand circular polarization \\
Receiver Gain & $4.5 \mathrm{~dB}$, Half-power beam width $=95^{\circ}[16]$ \\
Atmospheric/Ionospheric Losses & -1 dB loss within $30^{\circ}$ of boresight \\
Signal Wavelength & Negligible $[10]$ \\
Distance between satellite and aircraft & 0.275 m $(1090 \mathrm{MHz})$ \\
\end{tabular}

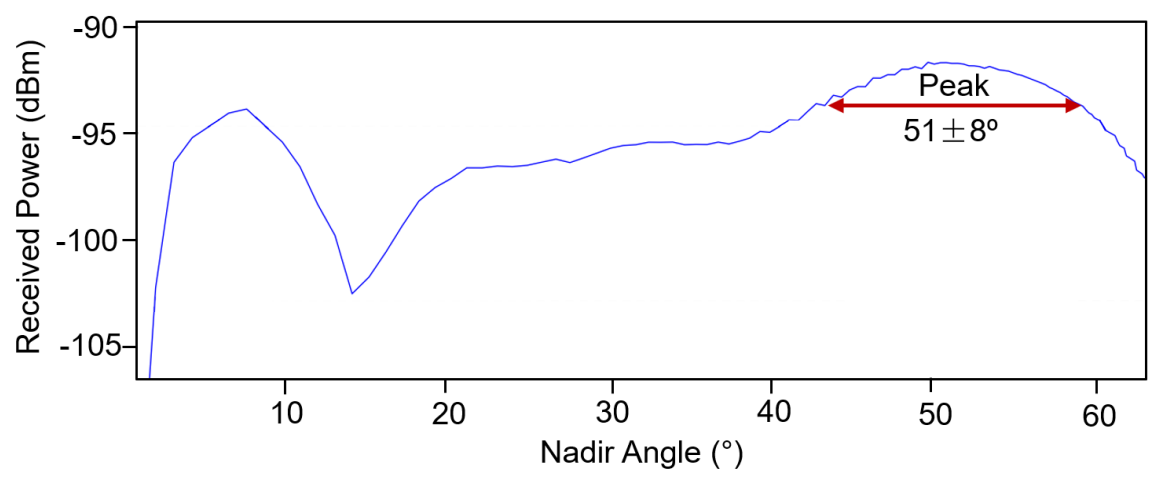

Figure 3. Modelled ADS-B received signal strength for CanX-7 in relation to degrees from satellite nadir for a $500 \mathrm{~W}$ transmitter and a typical aircraft radiation pattern [10]. The strongest signal occurs at a nadir angle of $51^{\circ} \pm 8^{\circ}$, which is the angle measured from the aircraft nadir to the aircraft.

Since larger nadir angles mean greater distance to the aircraft, this implies that transmitter gain is highest in this region. While the satellite receiver pattern is relatively constant over a large area, the gain is greatest within $30^{\circ}$ of the boresight and moves with respect to nadir as a result of the magnetic torquer aligning with the north magnetic pole. Most aircraft detections were north-northwest of the satellite as it transited through the AO (Figure 4), implying that messages were generally received ahead of the spacecraft on ascending passes and behind 
(a)

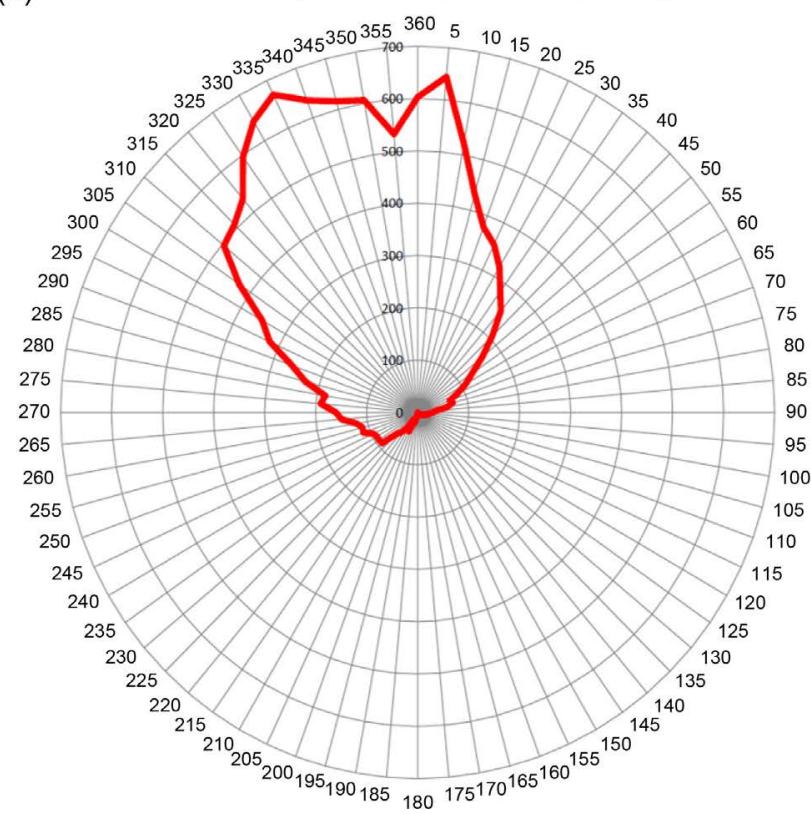

(b)

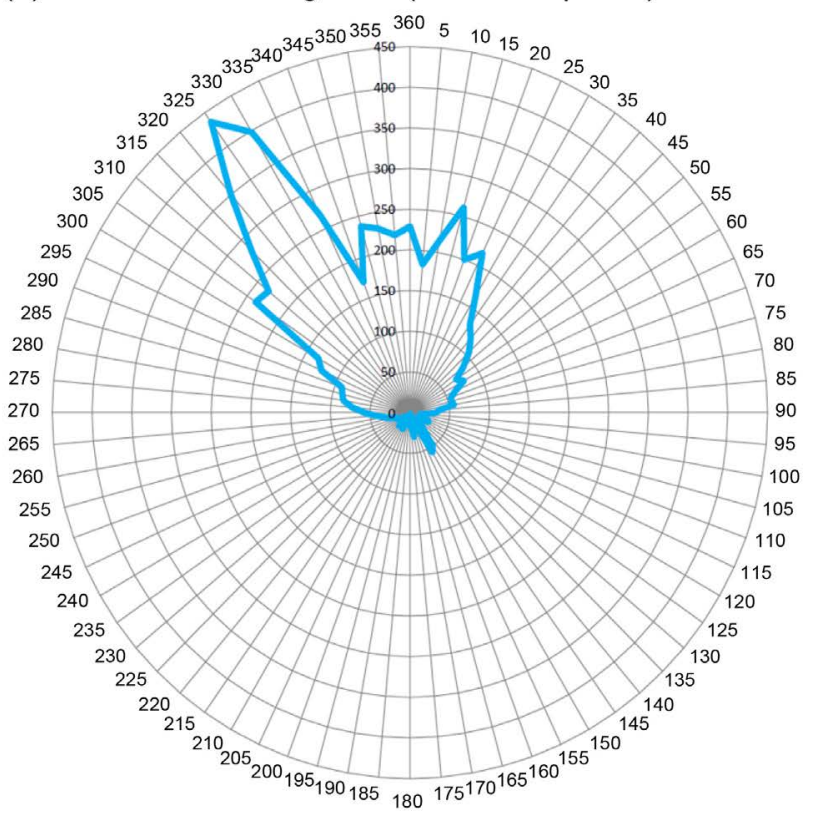

Figure 4. Polar plot of CanX-7 radiation pattern for (a) descending and (b) ascending passes. Each point on the respective outline is established by the overall number of messages (vertical axis) received at that azimuth angle in relation to the spacecraft. The north-northwest bias is a function of the satellite magnetic torquer attitude control system. The ascending pass pattern is notably less ordered than the descending pass, which may be a function of fewer data points or an issue with the magnetic torquer as the satellite moves northward.

the spacecraft on descending passes [15].

A Received Signal Strength Indicator (RSSI) value between 0 and 255 was assigned to ADS-B signals received by CanX-7. For this study, $95.8 \%$ of the received signals fell below a RSSI value of 40 , which is close to the Minimum Detectable Signal (MDS) for the payload (Figure 5). Analysis of consecutive messages originating from the same aircraft commonly showed widely varying RSSIs in the 0 to 40 range, suggesting that values near the noise floor may not always be accurate. The low signal strength led to a wide range of contact continuity, from single transmission receptions to hundreds of near continuous messages [13]. The proximity of the received signal strength to the receiver MDS allowed for an analysis of optimal aircraft-satellite orientation for ADS-B transmission detection. This was accomplished by first determining the position of the satellite at the time of signal reception and then the location of the transmitting aircraft. Satellite position was determined with the most appropriate two-line element set, while aircraft GPS location is encoded on the ADS-B position message. The angle between the antenna boresight and the aircraft was determined by assuming the antenna was aligned with the Earth's magnetic field using the International Geomagnetic Reference Field (IGRF). Nadir and boresight angles, as well as range to the aircraft, were determined using STK in conjunction with MATLAB. Figure 6 shows the geometry of the satellite and aircraft and relevant parameters. 


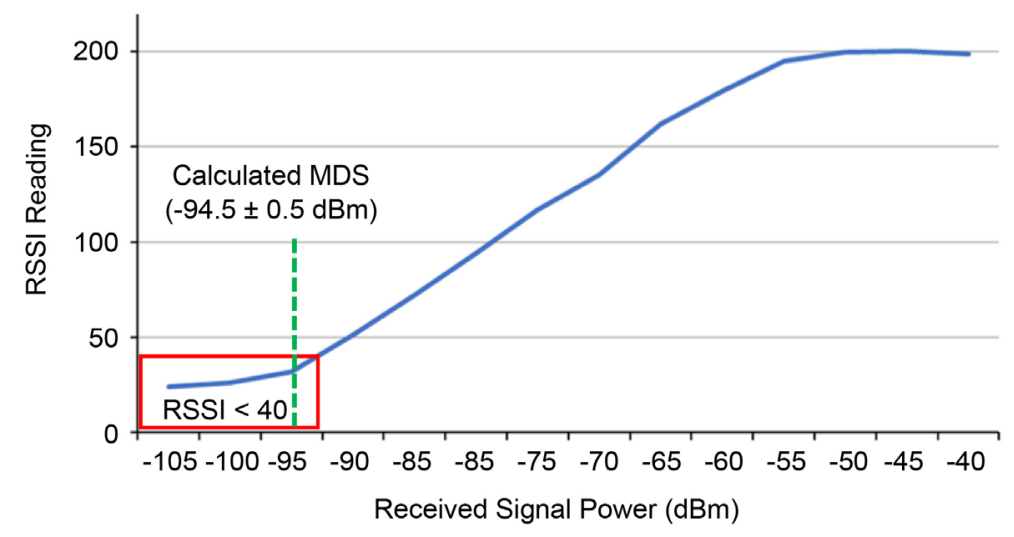

Figure 5. RSSI versus received signal power for the CanX-7 ADS-B payload. For this study, $95.8 \%$ of the received signals were close to the receiver MDS with an RSSI less than 40. Low RSSI values commonly showed high variability between consecutive messages originating from the same aircraft, suggesting potential inaccuracies for these values.

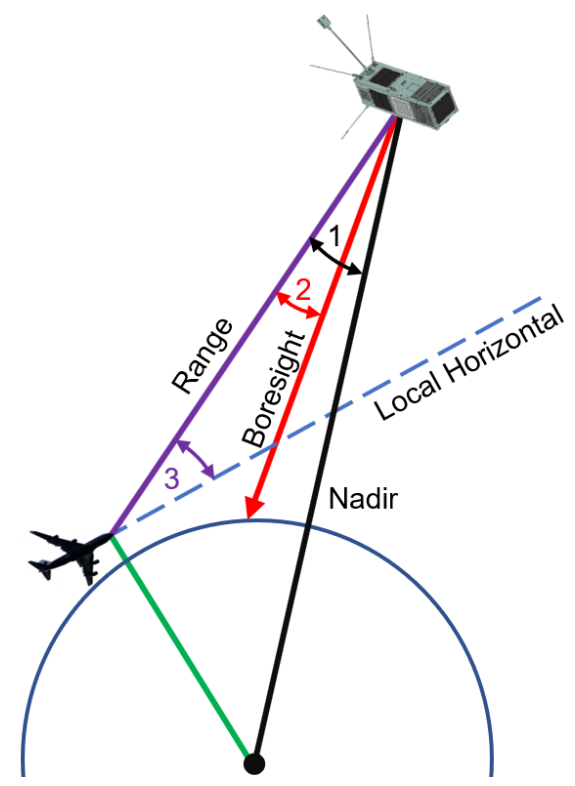

Figure 6. The determination of aircraft and satellite position at a specific time allows the calculation of range. The calculation of nadir 1) and boresight 2) angles based on elevation angle 3) relative to the local horizontal of the aircraft was determined through vector analysis. An elevation angle of zero degrees results in a maximum nadir angle of $64.4^{\circ}$ and a range of approximately $3100 \mathrm{~km}$.

\section{Results}

\subsection{Ascending/Descending Pass Disparities}

A significant contrast was observed in nadir angles for descending and ascending passes (Figure 7). The average nadir angle measured for descending passes was $50.1^{\circ}$ with a standard deviation of $7.9^{\circ}$. These values compare favorably with the ADS-B propagation model, which predicts the strongest aircraft signals 


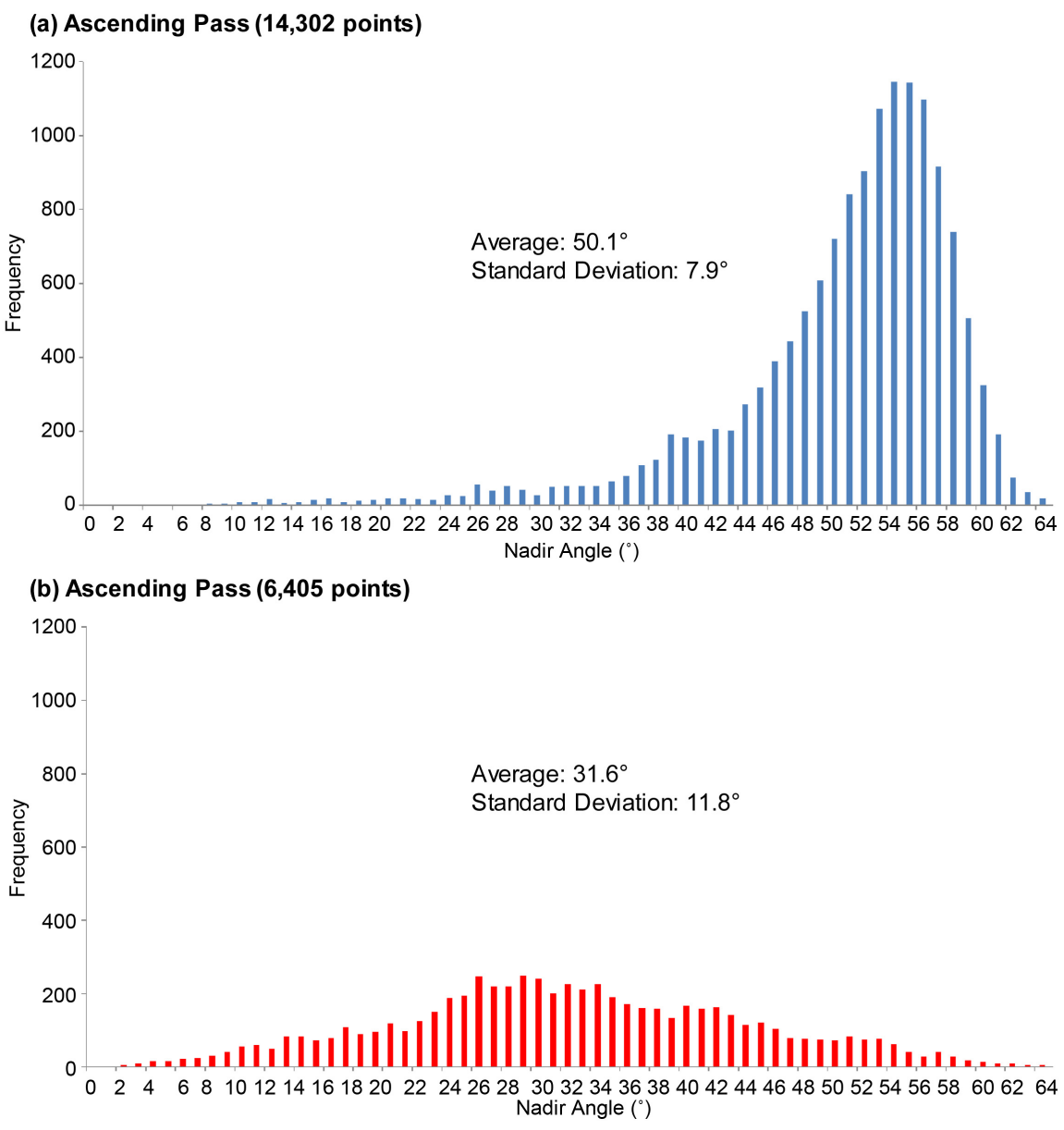

Figure 7. Histograms are shown of the CanX-7 nadir angle detections using one-degree bins for (a) descending and (b) ascending passes in the AO. There is a significant contrast in the distribution of nadir angles for the two passes. The descending pass nadir angles are consistent with the peak of the signal propagation model (Figure 3), while the ascending pass does not reflect the model.

between $43^{\circ}$ and $59^{\circ}$ [10]. For descending passes, $81.3 \%$ of the received messages were in this range with $90.0 \%$ of the messages having a nadir angle greater than $40^{\circ}$. In contrast the ascending passes had an average nadir angle of $31.6^{\circ}$ with a standard deviation of $11.8^{\circ}$. Only $16.9 \%$ of the contacts fell between $43^{\circ}$ and $59^{\circ}$, while $24.8 \%$ exceeded $40^{\circ}$. Sensitivity to latitude and longitude was investigated to determine if these parameters influenced the nadir angle results. The distribution of nadir angle detections showed no significant change for descending and ascending passes respectively as a function of latitude or longitude, although more variability was noted for the ascending pass. An investigation of nadir angle as a function of aircraft direction of travel also revealed no change in the distribution of nadir angles for either type of pass. A comparison of boresight angles between the two passes showed a considerable difference (Figure 8). While the average boresight angle for descending passes $\left(27.2^{\circ}\right)$ and ascending passes $\left(32.6^{\circ}\right)$ are comparable, the descending pass values have a much lower standard deviation $\left(7.4^{\circ}\right)$ than the ascending passes $\left(16.5^{\circ}\right)$. The difference between nadir 


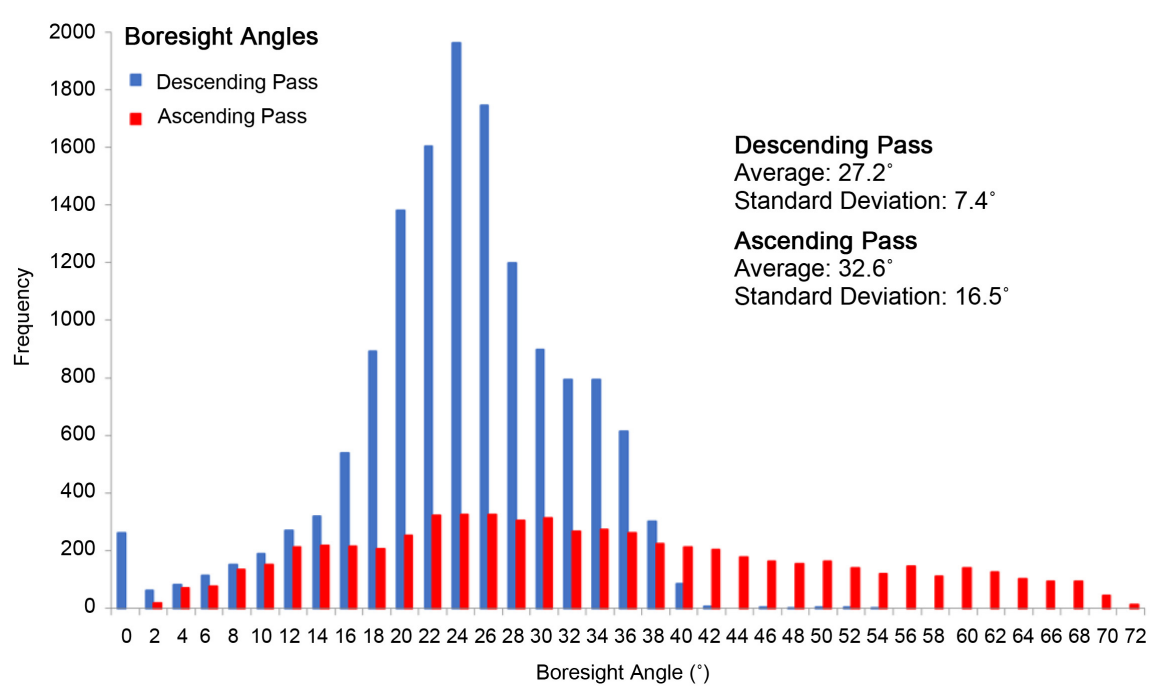

Figure 8. A comparison of boresight angles for descending and ascending passes shows markedly different distributions. Descending passes have a relatively tight grouping centered around $24^{\circ}$, while the ascending passes are spread across the spectrum with values ranging up to $72^{\circ}$.

and boresight angles are also markedly different for the two types of passes (Figure 9). There is a significant number of contacts that have boresight angles less than the nadir angle (58.2\%), while $98.2 \%$ of the descending pass contacts exhibit nadir angles greater than the boresight angle with a strong peak centered at $24^{\circ}$.

The peak of descending pass nadir angles centered at approximately $55^{\circ}$ (Figure $7(\mathrm{a})$ ) can be explained in terms of the signal propagation model and the observed averages for nadir and boresight angles. Figure 10 shows CanX-7 with the ADS-B antenna boresight aligned with the north magnetic pole. A $\pm 30^{\circ}$ beam on either side of the boresight is shown since this represents the highest gain of the ADS-B receiver. In this scenario, contact is attained if the strongest signal from the aircraft as predicted by the signal propagation model falls within the $\pm 30^{\circ}$ beam of the antenna.

There is a fundamental difference in the observed nadir angles for descending and ascending passes. Although there are 55\% less data points for the ascending passes, there is enough to produce a trend that should mirror the descending passes. A comparison between descending and ascending pass for the same airframe (Figure 11) produced dissimilar results that reflected the trend observed in Figure 7 with lower nadir angles observed on the ascending pass. Given that the payload performance is constant, a potential difference between the two passes is the behavior of the magnetic torquer. For descending passes the satellite is close to the north magnetic pole before entering the AO, which should produce a stable platform. For ascending passes the satellite approaches from the equator where the Earth's magnetic field is weaker and parallel to the surface. As the satellite moves northward the satellite slews toward the north magnetic pole. There is evidence in the dataset that suggests the satellite boresight is not always 


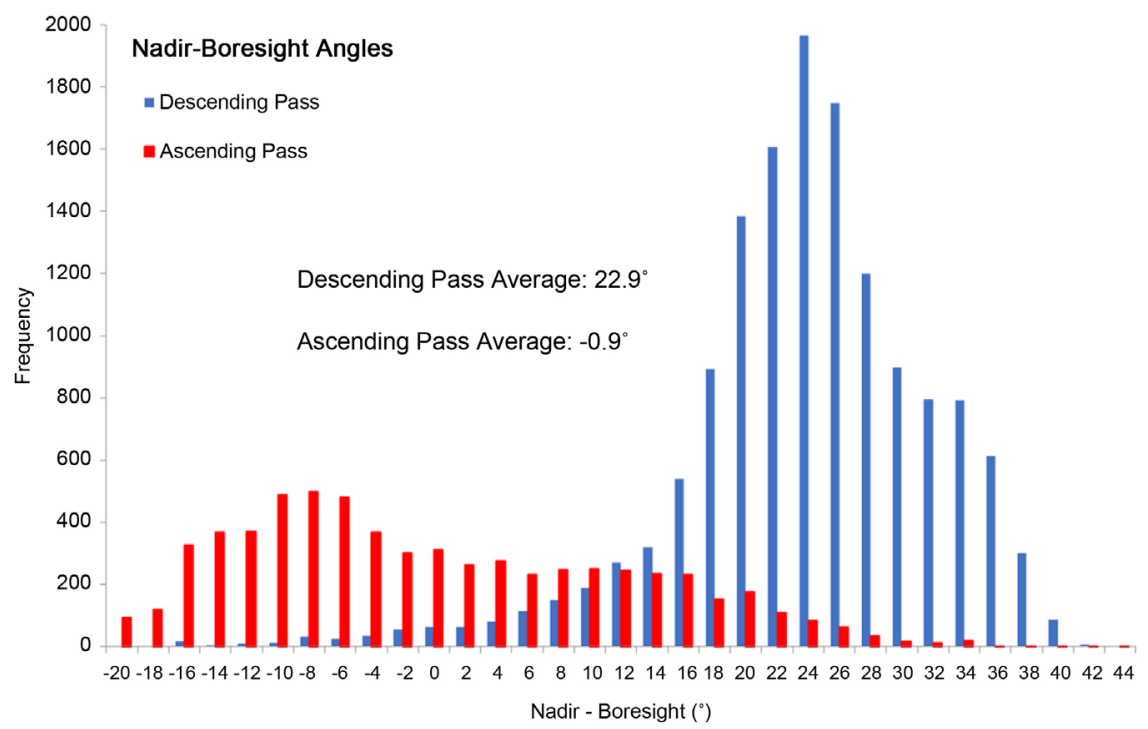

Figure 9. A comparison of nadir minus boresight angles is shown for descending and ascending passes. Ascending passes are more variable with an overall negative value, while descending passes are $98.2 \%$ positive with a strong peak between $20^{\circ}$ and $30^{\circ}$.

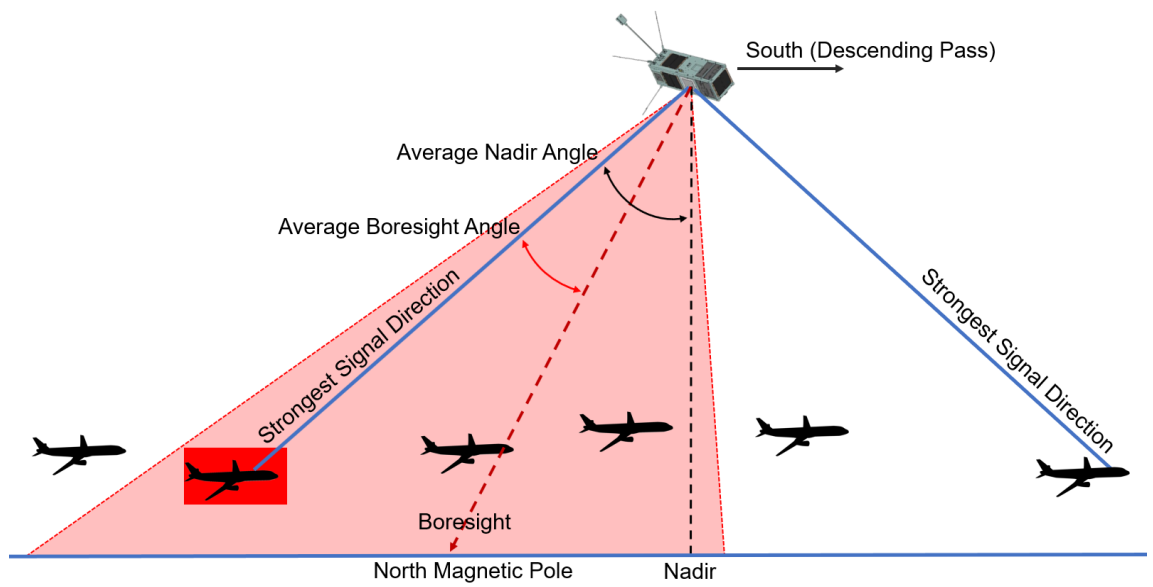

Figure 10. A schematic is shown for a descending pass to illustrate the distribution of nadir angles observed in Figure 7(a). The strongest signal direction relative to satellite nadir as predicted by propagation model is indicated in blue. The CanX-7 radiation pattern is shown in light red $\left( \pm 30^{\circ}\right.$ beam) with the boresight aligned with the north magnetic pole. The CanX-7 radiation pattern and the preferred signal direction must line up for contact. Only the aircraft highlighted in red is in contact. Aircraft to the right in the diagram will be detected as CanX-7 moves southward. Contact closer to nadir can occur, but the schematic describes the dominant trend in the data.

aligned with the north magnetic pole during the northward transit through the AO.

1) Re-creations of passes with STK shows contact a significant distance behind the satellite as it approaches the $\mathrm{AO}$ from the south, implying that the satellite is not aligned with the magnetic field. The detection behind the behind the satellite as it moved northward was also observed at times within the $\mathrm{AO}$ as shown in Figure 4(b). 


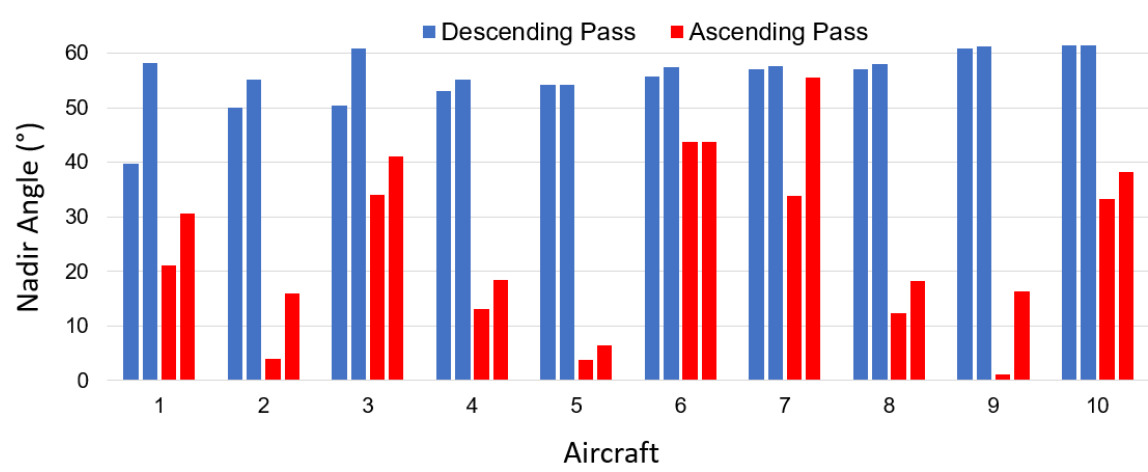

Figure 11. The minimum and maximum nadir angle is shown for ten identical airframes for ascending and descending passes. The data suggests that the satellite is behaving differently for the two passes.

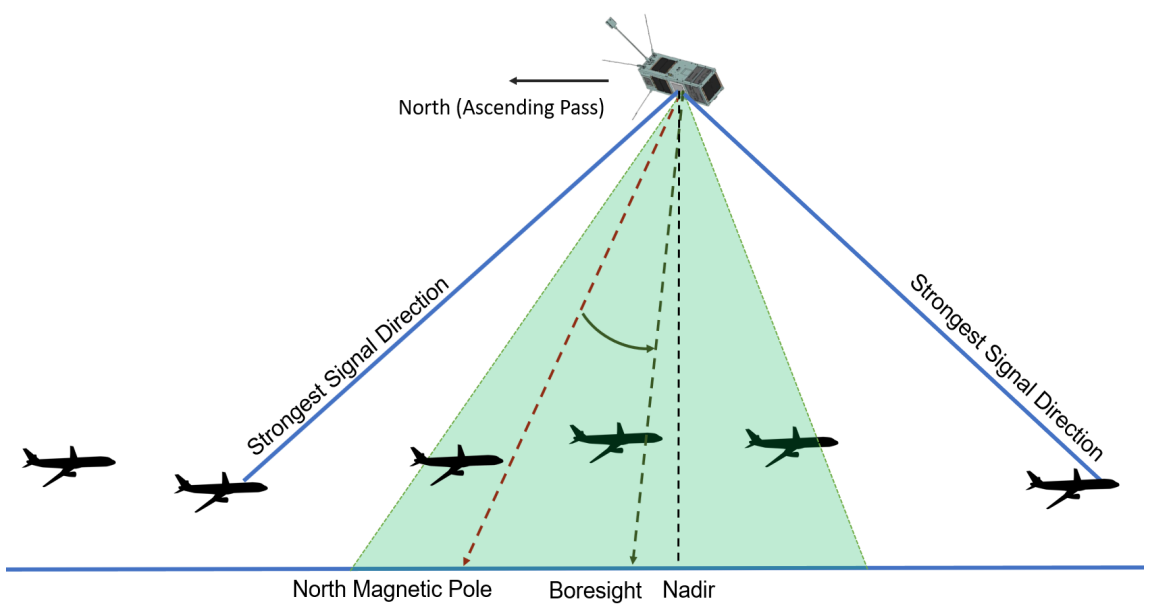

Figure 12. A schematic is shown for an ascending pass. If the boresight is pointing closer to nadir than predicted, then contacts with nadir angles related to the strongest signal direction will not be in the $\pm 30^{\circ}$ beam of the ADS-B receiver. The significantly reduced range to aircraft, particularly along the boresight, may allow weaker signals to be detected that would otherwise be missed with a more inclined boresight. An oscillating boresight could give a variety of different nadir angles as observed in Figure 7(b).

2) The extreme range of boresight angles (Figure 8) suggests an inaccuracy in the modeled alignment since the receiver gain is reduced by up to $3 \mathrm{~dB}$ at the outer edges of the beam.

3) For ascending passes the average boresight and nadir angle are almost equal, which should result in a greater number of low nadir angles than seen in Figure $7(\mathrm{~b})$. This provides further evidence that the boresight is not aligned with the north magnetic pole.

Since all nadir angles are based on known quantities and assumed to be correct, the evidence suggests that the satellite is pointing closer to nadir than predicted for ascending passes. The difference in average nadir angles indicate aircraft are $27 \%$ closer to the satellite for ascending passes (887 km average range) than descending passes (1216 km average range). The reduction in range has a significant impact on the received power since it is inversely proportional to the 
square of the distance between the satellite and aircraft (Equation (1)). Figure 12 shows a scenario in which the satellite is pointing closer to nadir than predicted by the IGRF. In this case the strongest signal direction does not fall within the $\pm 30^{\circ}$ beam, which would explain the lack of contacts for nadir angles greater than $50^{\circ}$. The reduced range to aircraft, particularly along the boresight which has the highest gain, would allow the ADS-B payload to receive signals from a range of smaller nadir angles. An oscillating boresight may produce a variety of nadir angles as seen Figure 7(b).

Although the RSSI value of a single message was considered potentially unreliable, the global statistics revealed a strong trend of weaker signals for the ascending passes when compared to descending passes (Figure 13). This supports the concept that the ADS-B receiver was not ideally oriented to receive the modeled strongest signals at nadir angles $51^{\circ} \pm 8^{\circ}$. Additionally, there was close correlation between received and calculated (Equation (1)) signal strength for descending passes, which was not evident for ascending passes. The reduced correlation between observed and calculated signal power for the ascending pass could be attributed to a pointing inaccuracy.

\subsection{Descending Pass Analysis}

Further analysis was carried out on the descending pass to determine if aircraft with many consecutive messages influenced the prominent peak observed in the data in Figure 7(a). The minimum and maximum nadir angle was recorded for 1,096 different contacts, which included 173 single-message occurrences (Figure 14). The results showed an average minimum to maximum nadir angle range of $47.9^{\circ}$ to $55.2^{\circ}$, with single-message contacts averaging $53.5^{\circ}$. The overall nadir

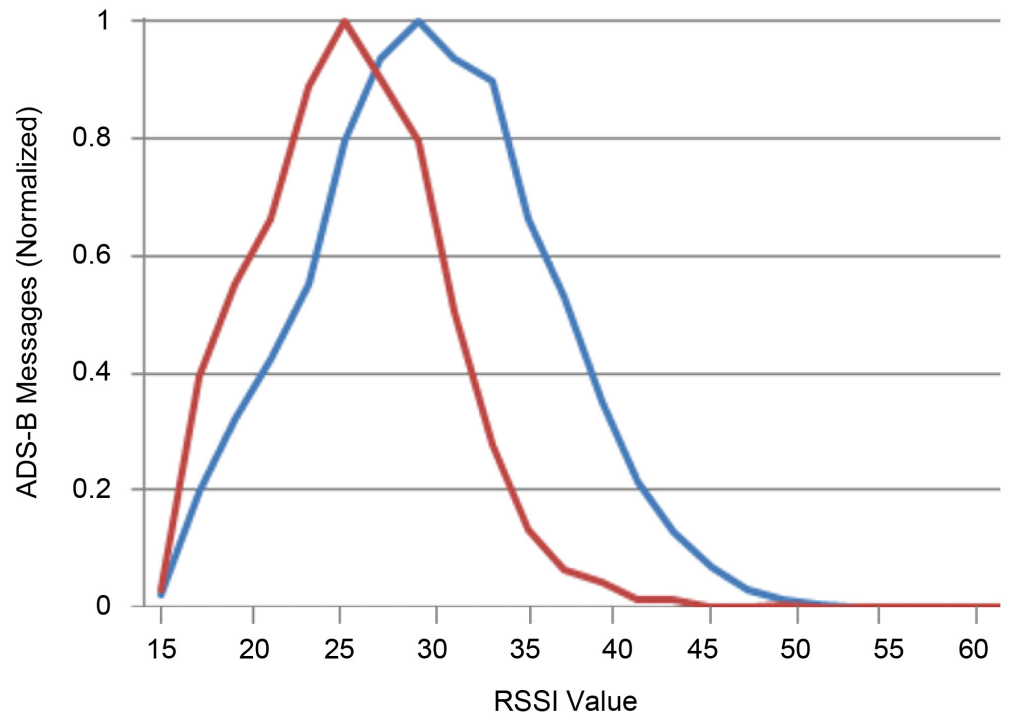

Figure 13. RSSI values for descending and ascending passes are shown. The weaker signals observed for the ascending passes could be attributable to the strongest signals not being detected due to the orientation of the satellite as shown in Figure 12. 


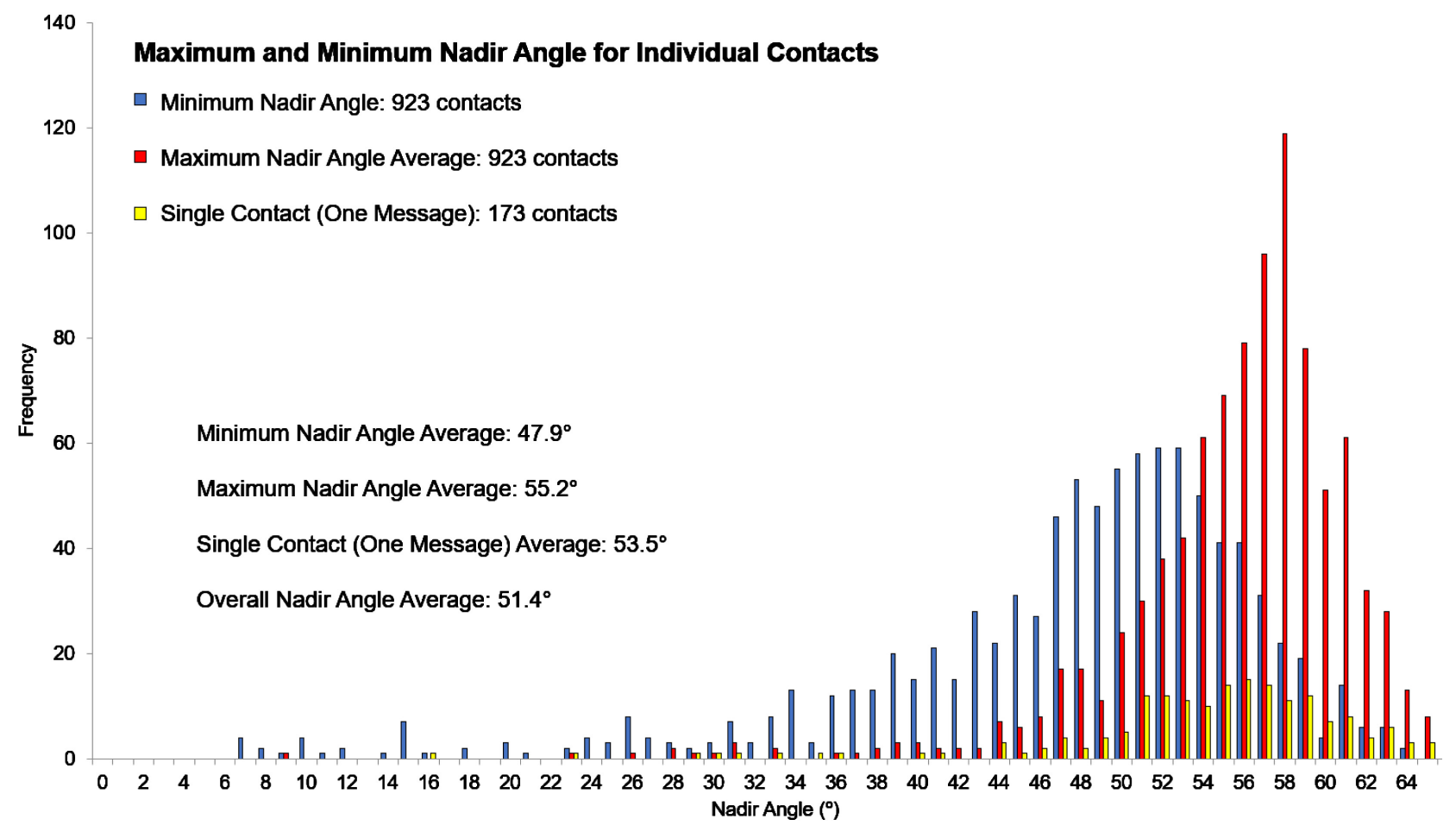

Figure 14. Minimum and maximum nadir angles are shown for 923 contacts for descending passes. Nadir angles for single-message contacts are also indicated. The results are similar to Figure 7(a), signifying that aircraft with many detected messages do not have a significant influence on the overall observed nadir angle distribution.

angle of $51.4^{\circ}$ is comparable the $50.1^{\circ}$ observed for all descending pass position messages. The increase in detections with respect to nadir angle is in part a function of geometry. As the nadir angle becomes larger there is a corresponding growth in area coverage. To allow direct comparison between different nadir angles, the descending pass data used to construct Figure 14 was calculated in terms of message detections per square kilometer and normalized (Figure 15). When contact density is taken into consideration, a peak closer to the nadir is revealed, while the highest values are at nadir angles between $45^{\circ}$ and $55^{\circ}$. If the signal propagation model from Figure 3 is superimposed on the aircraft message density histogram, the peaks from both graphs coincide (Figure 15). A similar process for ascending passes is not included, since the evidence indicates that the beam is not consistently covering all possible nadir angles as illustrated in Figure 12 .

\section{Summary and Conclusions}

An analysis of CanX-7 ADS-B position data collected over the Gander and Shanwick OCAs revealed a disparity in the distribution of nadir angles between descending and ascending passes of the satellite. For descending passes, the average nadir angle was $50.2^{\circ}$ with over $90 \%$ of the contacts exhibiting nadir angles in excess of $40^{\circ}$. The abundance of large nadir angles is in keeping with the ADS-B signal propagation model in which the strongest signals are predicted to be at nadir angles ranging from $43^{\circ}$ to $59^{\circ}$. With respect to the CanX-7 ADS-B 


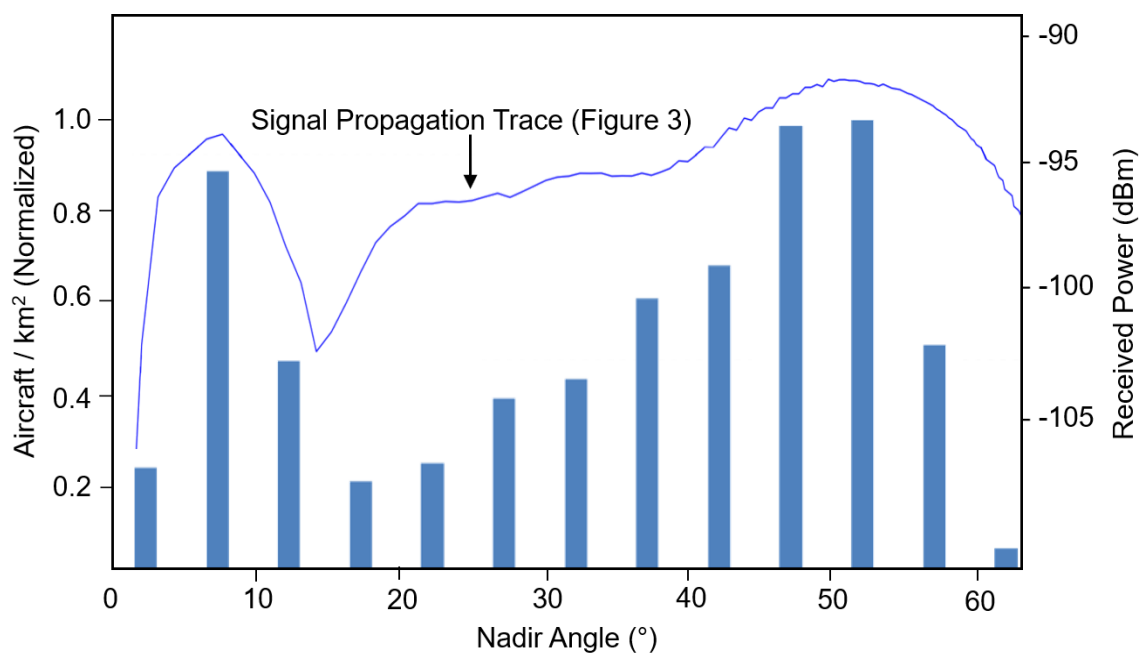

Figure 15. Message density per square kilometer in $5^{\circ}$ bins based on minimum and maximum nadir angle is compared to the signal propagation model shown in Figure 3. There is a good correlation between observation and the model.

payload this is relevant since the received signals were close the minimum detection threshold of the sensor. In contrast, the ascending passes averaged $31.6^{\circ}$ for nadir angles and showed a broader distribution than seen in the descending pass data. The evidence suggests that the satellite magnetic torquer was not always aligned with the north magnetic pole as it approached from the south and transited through the $\mathrm{AO}$, resulting in ascending pass nadir angles that were not reflective of the full range of values. When descending pass nadir angles were grouped into minimum and maximum values for each individual contact and evaluated in terms of messages per square kilometer, there was good agreement with the ADS-B signal propagation model.

The CanX-7 ADS-B mission demonstrated that nadir angle plays an important role in the reception of signals. For a nadir pointing spacecraft, it is possible that the strongest signals at high nadir angles will not be in the main beam of the receiving antenna. For aircraft with transmitters less than $500 \mathrm{~W}$, this could potentially be a problem for operational space-based ADS-B systems. The Iridium NEXT constellation will provide worldwide coverage of aircraft from space, but for air traffic control purposes there must be reliable reception of ADS-B signals from all aircraft. This study supports the use of a modified upper aircraft ADS-B antenna for space applications, one that is more omnidirectional than the current quarter wave monopole.

\section{Conflicts of Interest}

The authors declare no conflicts of interest regarding the publication of this paper.

\section{References}

[1] RTCA DO-260B (2009) Minimum Operational Performance Standards for 1090 
MHz Extended Squitter Automatic Dependent Surveillance-Broadcast (ADS-B) and Traffic Information Services-Broadcast (TIS-B). Radio Technical Commission for Aeronautics.

[2] Aerion Press Release (2019) Aireon System Goes Live-Trial Operations Begin Over the North Atlantic Marking New Chapter in Aviation History. https://aireon.com/2019/04/02/aireon-system-goes-live-trial-operations-begin-nort h-atlantic-marking-new-chapter-aviation-history/

[3] Francis, R., Vincent, R. Noël, J.M., Tremblay, P., Desjardins, D., Cushley, A. and Wallace, M. (2011) The Flying Laboratory for the Observation of ADS-B Signals. International Journal of Navigation and Observation, 2011, Article ID: 973656. https://doi.org/10.1155/2011/973656

[4] Vincent, R.F. (2011) The FLOAT Mission: Promoting Teamwork in the Classroom. Physics in Canada, 67, No. 4.

[5] Cushley, A. and Noël, J.M. (2014) Ionospheric Tomography Using ADS-B Signals. Radio Science, 49, 549-563.

[6] Cushley, A.C., Kabin, K. and Noël, J.M. (2017) Faraday Rotation of Automatic Dependent Surveillance-Broadcast (ADS-B) Signals as a Method of Ionospheric Characterization. Radio Science, 52, 1293-1300. https://doi.org/10.1002/2017RS006319

[7] Cushley, A.C., Kabin, K. and Noël, J.M. (2018) Faraday Rotation, Total Electron Content, and Their Sensitivity to the Average Parallel Component of the Magnetic Field. Radio Science, 53, 1075-1088. https://doi.org/10.1029/2018RS006667

[8] Francis, R. Noël, J. and Vincent, R. (2011) Orbital Monitoring of Automatic Dependent Surveillance-Broadcast (ADS-B) Signals for Improved Air Traffic Surveillance in Remote and Oceanic Airspace. Proceedings of the 62nd International Astronautical Congress, Cape Town, 3-7 October 2011.

[9] Van Der Pryt, R. and Vincent, R. (2015) A Simulation of Signal Collisions over the North Atlantic for a Spaceborne ADS-B Using Aloha Protocol. Positioning, 6, 23-31. https://doi.org/10.4236/pos.2015.63003

[10] Van Der Pryt, R. and Vincent, R. (2015) A Simulation of the Reception of Automatic Dependent Surveillance-Broadcast (ADS-B) Signals in Low Earth Orbit. International Journal of Navigation and Observation, 2015, Article ID: 567604. https://doi.org/10.1155/2015/567604

[11] Van Der Pryt, R. and Vincent, R. (2016) A Simulation of Reflected ADS-B Signals over the North Atlantic for a Space-Borne Receiver. Positioning, 7, 51-62. https://doi.org/10.4236/pos.2016.71005

[12] Vincent, R. (2015) The CanX-7 ADS-B Mission: Tracking Aircraft from Space. Canadian Space Society: Canadian Space Summit, Vancouver, 19-20 November 2015, $1-12$.

[13] Vincent, R. and Van Der Pryt, R. (2015) The CanX-7 Nanosatellite ADS-B Mission: A Preliminary Assessment. Positioning, 8, 1-11. https://doi.org/10.4236/pos.2017.81001

[14] University of Toronto Institute of Aerospace Studies-Space Flight Laboratory, Nanosatellites: CanX-7. https://www.utias-sfl.net/?page_id=210

[15] Freitag. K. (2017) An Analysis of Automatic Dependent Surveillance-Broadcast Signals Received by the Canx-7 Nanosatellite: Examining the North Atlantic Oceanic Airspace Control Area. M.Sc. Thesis, Royal Military College of Canada.

[16] Bennett, I., Paris, A., Cotton, B. and Zee, R. (2016) Nanosatellite Aircraft Tracking: Simulation and Design of the CanX-7 ADS-B. The Canadian SmallSat Conference, Toronto, 2-3 February 2016. 